\title{
GIF NATURAL RADIOCARBON MEASUREMENTS V
}

\author{
G. DELIBRIAS, M. T. GUILLIER, and J. LABEYRIE
}

Centre des Faibles Radioactivités, Centre National de la Recherche Scientifique, (91) Gif-sur-Yvette, France

The list given below contains the $\mathrm{C}^{14}$ dates obtained for only archaeologic samples, during 1966 and 1967; for the study of the geologic samples dated during the same period, cf. our previous list Gif-III (Radiocarbon, 1969, v. 11, p. 327-344). The same instruments and techniques have been employed.

Dates were calculated on the basis of a $\mathrm{C}^{14}$ half-life of $5568 \mathrm{yr}$ and $95 \%$ of NBS oxalic acid as a modern standard.

\section{ACKNOWLEDGMENTS}

We should like to thank M. Rousseau for routine sample preparation and J. P. Garnier for assistance with electronic equipment.

\section{SAMPLE DESCRIPTIONS}

I. FRANCE

\section{A. Southwest France}

\section{B.C.}

Charcoal from circular hearth, Saliés-de-Béarn $\left(43^{\circ} 28^{\prime} \mathrm{N}\right.$ Lat, $0^{\circ}$ $55^{\prime}$ W Long), Basses Pyrénées; found with potsherds similar to others of sites of Late Bronze Age, in Aquitaine. Coll. by M. Gauthier and subm. 1965 by J. Coupry, Dir. des Antiquités Historiques d'Aquitaine, Bordeaux. Comment: these circular hearths were used for industrial extraction of salt. Result confirms Late Bronze age indicated by pottery.

\section{Gif-395. Saint-Martin de Caralp, Ariège}

$9150 \pm 1000$

7200 B.c.

Bones of fossil reindeer (Rangifer tarandus) in a stalagmitic layer in the Bernard Cave, at Saint-Martin de Caralp ( $43^{\circ}$ N Lat, $1^{\circ} 32^{\prime}$ E Long), Ariège. Coll. and subm. 1965 by J. Bouchud, CNRS, Paris. Comment: extracted collagen was quite short and diluted with "dead" $\mathrm{CO}^{2}$ to bring sample to usual counter pressure. Date not acceptable for fossil reindeer which, according to submitter, definitely left France ca. 12,000 B.P. (Bouchud, 1964).

\section{Gif-376. Lectoure, Gers}

Charcoal from pottery kilns, at Lectoure $\left(43^{\circ} 56^{\prime} \mathrm{N}\right.$ Lat, $0^{\circ} 38^{\prime} \mathrm{E}$ Long), Gers. Some kgms of Roman coins have been found in each one. Coll. by M. Larrieu and subm. 1965 by E. Thellier, Inst. de Physique du 
Globe, Paris. Comment: dated from Late Roman period by coins and by average magnetic measurements obtained on the burnt clay of 4 kilns. Either $\mathrm{C}^{14}$ date is aberrant or sample is not representative.

\section{Gif-322. Bordeaux, Gironde}

Wood from piles extracted during foundation diggings in the city of Bordeaux (44 $50^{\prime} \mathrm{N}$ Lat, $0^{\circ} 34^{\prime} \mathrm{W}$ Long), Gironde. Coll. and subm. 1964 by B. Vermeylen, Soc. Archéolog. de Bordeaux. Comment: because of the source of sampling, depth is difficult to determine; but piles were probably under a mosaic level, dated 4th century A.D.; give earlier date than believed for foundation of city, which probably was, at first, a town built on pilings.

\section{Gif-332. Chassang, Chamboulive, Corrèze}

\section{A.D. 1490}

$460 \pm 100$

Charcoal from a souterrain at Chassang, Chamboulive $\left(48^{\circ} 26^{\prime} \mathrm{N}\right.$ Lat, $1^{\circ} 42^{\prime} \mathrm{E}$ Long), Corrèze. Coll. and subm. 1964 by H. Boudrie, Uzerche, Corrèze. Comment: this kind of building, which is found in different French regions, seems to have been not only a refuge during disturbed times of history, but also a cultural site.

\section{Gif-392. Faycelles, Lot}

$$
1420 \pm 120
$$

Human bones from tombs found in barrow at Faycelles $\left(44^{\circ} 34^{\prime}\right.$ N Lat, $1^{\circ} 59^{\prime}$ E Long), Lot. Coll. and subm. 1965 by P. Delbos, Caussade, Tarn et Garonne. Comment: tombs seem to have been sunk in the barrow, which is surely older. Assoc. pottery is very rough and its age could not be appreciated exactly. This necropolis was believed to be from a Pagan, isolated people in the Merovingian age; date agrees with this interpretation.

\section{Gif-212. Grotte de Saint-Géry, Loze, $\quad 1060 \pm 120$ Tarn et Garonne A.D. 890}

Charcoal of archaeologic layer of the Grotte de Saint-Géry $\left(44^{\circ} 30^{\prime}\right.$ $\mathrm{N}$ Lat, $1^{\circ} 35^{\prime} \mathrm{E}$ Long), Loze, Tarn et Garonne. Coll. 1961 and subm. 1963 by A. Cavaillé, Mus. d'Histoire Nat. de Montauban. Comment: does not agree with archaeologic data; construction of a wall through the layer can explain pollution due to the introduction of more recent charcoals. Expected age: la Tène II Iron age.

\section{Gif-442. Grotte des Cascades, Creissels, Aveyron 1320 в.c. \\ $3270 \pm 150$}

Charcoal from a Chalcolithic level in the Grotte des Cascades, Creis-

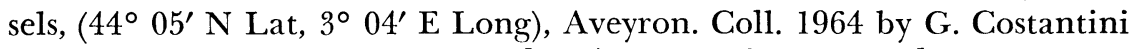
and subm. 1966 by J. Maury, Rodez, Aveyron. Comment: does not agree very satifactorily with the well-dated Chalcolithic furniture found in site (Costantini, 1965). Expected age: 2000 to 2200 B.c. 
Grotte de Sargel series, Saint-Romé de Cernon, Aveyron

Charcoals from archaeologic levels of the Grotte de Sargel, SaintRome de Cernon (44 $01^{\prime}$ N Lat, $2^{\circ} 57^{\prime}$ E Long), Aveyron (Soutou, 1966). Coll. 1965 and subm. 1966 by J. Maury and A. Soutou.

\section{Gif-444. Grotte I de Sargel, Level VI}

$4500 \pm 150$

Level VI, with transition between Chassean and early Chalcolithic industries.

Gif-445. Grotte I de Sargel, Level $X$

$4570 \pm 150$

Level X, with pure Chassean Neolithic industry.

2620 B.C.

Gif-328. Grotte I de Sargel

$3710 \pm 180$

1760 B.c.

From Chalcolithic level of Cave I, Sargel. Comment: fits very well with type of pottery assoc.

General Comment: corresponds nicely to expected ages.

\section{Gif-443. Grotte des Salzets, Mostuéjouls, Aveyron}

$8770 \pm 200$

Charcoal from a unique archaeologic level, $30 \mathrm{~cm}$ thick, in the Cave of Salzets, Mostuéjouls (44 $13^{\prime} \mathrm{N}$ Lat, $3^{\circ} 09^{\prime} \mathrm{E}$ Long), Aveyron. Coll. 1964 and subm. 1966 by J. Maury. Comment: agrees with geometric microlithic industry and a fauna with a primitive ox-deer found in the cave (Maury and Lacas, 1965). Similar date obtained for Rouffignac considered classic Sauveterrian site.

\section{Gif-446. Grotte de Puechmargues, La Roque- $\quad 6420 \pm 180$ Sainte-Marguerite, Aveyron \\ 4470 B.c.}

Charcoal found under a Chalcolithic ossuary in a Tardenoisian level, Grotte de Puechmargues, La Roque-Sainte-Marguerite $\left(44^{\circ} 08^{\prime} \mathrm{N}\right.$ Lat, $3^{\circ} 13^{\prime}$ E Long), Aveyron (Maury and Lacas, 1965). Coll. 1964 and subm. 1966 by J. Maury. Comment: younger date expected because of Neolithic affinities of tools and presence of pottery. Date is reasonable if compared to dates obtained for early Mediterranean Neolithic, ca. 4500 в.c.

Gif-331. Causse de Méjean, Gorges du Tarn, Lozère

$$
760 \pm 100
$$

Charcoal from grave in cave of Causse of Méjean, Gorges du Tarn (44 14' N Lat, 3 12' E Long), Lozère. Coll. and subm. 1964 by Y. Legoux, Bulles, Oise. Comment: not Neolithic as expected.

Gif-219. Mas Saint-Chely, Lozère

Charcoal from top layer of rectangular dolmen at Mas Saint-Chely

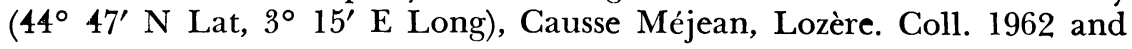


subm. 1963 by M. Lorblanchet, Montpellier, Hérault. Comment: indicates some re-utilization during Early Iron age of this Neolithic monument, frequent episode in this part of France.

\section{B. South, Southeast, and central France}

\section{Palaggiu, Sartene series, Corsica}

Charcoals from megalithic site Pallagiu, Sartene $\left(41^{\circ} 33^{\prime} \mathrm{N}\right.$ Lat, $8^{\circ}$ 53' E Long) Corsica (Grosjean, 1967). Coll. 1965 and subm. 1966 by R. Grosjean, Centre de Préhistoire Corse, CNRS, Paris.

Gif-476. Palaggiu, Corsica 1-1965

$$
2650 \pm 150
$$

From Funeral Chest A of the alignment of menhirs. Inferior level.

\section{Gif-477. Palaggiu, Corsica 2-1965}

$2680 \pm 150$

From a burning level, at foot of $\mathrm{N}$ alignment of menhirs.

General Comment: 1000 yr younger than expected. May not date site.

\section{Castello d'Alo, Bilia series, Corsica}

Charcoal from Torrean monument of complex site of Castello d'Alo, Bilia (41ํ $37^{\prime} \mathrm{N}$ Lat, $8^{\circ} 54^{\prime}$ E Long), Corsica (Grosjean, 1966). Coll. 1965 and subm. 1966 by R. Grosjean.

\section{Gif-478. Castello d'Alo, Corsica 3-1965}

$3100 \pm 110$

From central inferior hearth, in E cultural monument.

Gif-479. Castello d'Alo, Corsica 4-1965

$3500 \pm 120$

1550 в.c.

From lower burning level, over pavement, in E cultural monument. Comment: dates last ritual utilization of monument.

\section{Gif-480. Castello d'Alo, Corsica, 5-1965}

$3820 \pm 200$

1870 в.c.

From main hearth of C. W. room, in O. monument. Comment: typical monument dated older than Torrean civilization. Similar to Gsy-94B: $3865 \pm 125$ (Radiocarbon, 1966, v. 8, p. 130), Gif-243: $3770 \pm$ 250 (Radiocarbon, 1966, v. 8, p. 86) for similar Torrean sites at Tappa, in Corsica, and in Sardinia.

\section{La "Grotte Murée" series, Montpezat, Basses Alpes}

Charcoal from la "Grotte Murée," Montpezat (43 45' N Lat, $6^{\circ}$ 15' E Long), Basses Alpes (Courtin 1963; C. H. Lagrand, 1962).

Gif-139. La "Grotte Murée", Layer 4

$4160 \pm 250$

Lightly sintered at the top. Coll. and subm. by C. H. Lagrand, CNRS, Marseille. 
Gif-157. La "Grotte Murée", Layer 5

Coll. and subm. 1962 by J. Courtin, CNRS, Marseille.

General Comment: Layer 6 dated at $3960 \pm 175$ B.P., Gsy-116 (Radiocarbon 1966, v. 8, 74-95). Archaeologic ages: Layer 4, Late Bronze age, Layer 5, Middle Bronze age, and Layer 6, Chalcolithic age. Dates do not agree with supposed stratigraphy: discrepancy cannot be explained by contamination alone.

\section{Gif-303. Grotte de Unang, Mallemont, Vaucluse}

$5225 \pm 300$ 3275 B.c.

Charcoal from bottom of Level 9 of Grotte de Unang, Mallemont (44 $03^{\prime} \mathrm{N}$ Lat, $5^{\circ} 09^{\prime} \mathrm{E}$ Long), Vaucluse. Coll. 1963 and subm. 1964 by M. Paccard, Velleron, Vaucluse. Comment: dates this level of Late Cardial Neolithic, according to stratigraphy.

\section{Gramari series, Methamis, Vaucluse}

Site discovered by chance during gravel exploitation on right bank of Nesque R. (44 $01^{\circ}$ N Lat, $5^{\circ} 13^{\prime}$ E Long), Vaucluse. Excavations uncovered a few campsites and showed a stratigraphy made complex by streaming, human reworking, and torrential drift (Paccard, 1965, 1966). Coll. 1963 and subm. 1964 by M. Paccard.

\section{Gif-262. Gramari, Level C3a}

$3420 \pm 200$

Upper level, containing alternated habitation and inundation layers.

\section{Gif-263. Gramari, Level C3b}

$5090 \pm 300$

Middle level.

\section{Gif-264. Gramari, Level C3c}

Lower level.

General Comment: with bones of wild horse and bouquetin, and Sauveterrian artifacts. The dates obtained from these samples are younger than expected.

\section{Gif-437. Bridge of Avignon, Vaucluse}

$$
1540 \pm 120
$$

\section{A.D. 410}

Fragment of wood under foundations of extreme pillar of SaintBenezet bridge at Avignon (43 $56^{\prime} \mathrm{N}$ Lat, $4^{\circ} 48^{\prime} \mathrm{E}$ Long), Vaucluse. Coll. and subm. 1965 by Centre de Travaux d'Avignon Vallabrèques, Avignon, Vaucluse. Comment: as expected, dates famous bridge of Avignon at Gallo-Roman period.

\section{Gif-452. Dolmen des Fades, Pépieux, Aude}

Charcoal found in Late Neolithic, Fades' Megalithic tomb, Pépieux

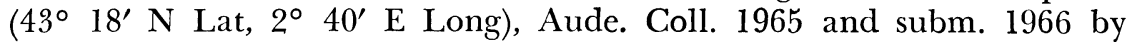


J. Guilaine, CNRS, Carcassonne. Comment: this site is known to have been used during Iron Age, Gallo-Roman period, and the Middle Ages.

Dates latest reutilization.

\section{Gif-453. Grotte de Gardouch, Beaucaire, Aude}

$7140 \pm 350$

Charcoal from sepulchral grotte de Gardouch, Beaucaire $\left(42^{\circ} 49^{\prime}\right.$ N Lat, $1^{\circ} 57^{\prime}$ E Long), Aude. Coll. 1965 and subm. 1966 by J. Guilaine. Comment: disagrees with expected Chalcolithic Late Bronze age. Submitter suggests possible utilization of old woods from the "Pinet" peat bog very near site.

Gif-483. Le Gaougnas, Cabrespine, Aude

$3160 \pm 200$ 1210 B.c.

Charcoal from Late Bronze age site of Le Gaougnas, Cabrespine ( $43^{\circ} 21^{\prime} \mathrm{N}$ Lat, $2^{\circ} 27^{\prime} \mathrm{E}$ Long), Aude. Coll. and subm. 1966 by J. Guilaine. Comment: in good range of dates for beginning of meridional Late Bronze age.

Gif-253. Cabane Giry, Nissan, Hérault

Charcoals of Kiln II, from La Cabane Giry, Nissan $\left(43^{\circ} 20^{\prime} \mathrm{N}\right.$ Lat, $3^{\circ} 02^{\prime}$ E Long), Hérault. Coll. 1963 by Abbé Giry and subm. 1964 by E. Thellier. Comment: dated for comparison with magnetic measurements of burnt clay from Kiln I located near Kiln II. Kilns seem uncontemporaneous.

Gif-450. Les Beaux-Escanin 2, Eyguières, $\quad 5000 \pm 250$ Bouches du Rhône

3050 B.C.

Charcoal from hearth from Les Beaux-Escanin, Eyguières $\left(43^{\circ} 45^{\prime}\right.$ $\mathrm{N}$ Lat, 4 $4^{\circ} 48^{\prime} \mathrm{E}$ Long), Bouches du Rhône. Coll. and subm. 1966 by R. Montjardin, Arles. Comment: assoc. with microliths and Chassean ceramics. Dates early provencal Chassean.

\section{Gif-451. Beaussement, Chauzon, Ardèche}

Charcoal from Level 3, Excavation 3, at Beaussement, Chauzon (44 $28^{\prime} \mathrm{N}$ Lat, 4 21' E Long), Ardèche. Coll. 1965 and subm. 1966 by R. Montjardin. Comment: correct date for Chalcolithic of Basse Ardèche (Montjardin, 1967).

\section{Gif-250. Le Pègue, Drôme}

$2130 \pm 150$

Charcoal, S 7 DIII, from Iron age archaeologic layer of le Pègue (44 $24^{\prime} \mathrm{N}$ Lat, $5^{\circ} 04^{\prime} \mathrm{E}$ Long), Drome. Coll. and subm. 1963 by $\mathrm{M}$. Samson, Paris. 
Charcoal from the Chalcolthic Level II A of "Abri du Capitaine," Sainte-Croix-de-Vernon ( $43^{\circ} 45^{\prime} \mathrm{N}$ Lat, $6^{\circ} 10^{\prime} \mathrm{E}$ Long), Basses Alpes. Coll. and subm. 1966 by J. Courtin, CNRS, Marseille. Comment: assoc. with beakers of "campaniform" provencal type.

\section{Avignon series, Vaucluse}

Charcoal from Quartier de la Balance sta., Avignon ( $43^{\circ} 56^{\prime} \mathrm{N}$ Lat, $4^{\circ} 48^{\prime}$ E Long), Vaucluse, where a complete stratigraphy was established from Chalcolithic age to present. Coll. and subm. 1966 by J. Courtin.

$4100 \pm 120$

Gif-705. Quartier de la Balance, Avignon, Level 32150 B.c. Chalcolithic level. $3500 \pm 120$
1550 B.c.

Under Level 3, same culture as 705 .

General Comment: Gif-705 gives correct age for this Late Neolithic level with "campaniform" beakers, but Gif-706 is contaminated.

\section{Gif-203. Roselet, Lac d'Annecy, Savoie}

$980 \pm 120$

Pile wood under $2 \mathrm{~m}$ of water in Lac d'Annecy at Roselet $\left(1^{\circ} 32^{\prime}\right.$ N Long, 45 49' E Lat). Coll. 1962 and subm. 1963 by M. Lamure, Paris. Comment: contemporary with foundation of Abbaye de Talloires and not of the Bronze period as first supposed.

Gif-274. Grotte de Chazelles, Ardèche $2730 \pm 150$ 780 B.C.

Charcoal from Level 519-536, Layer IV, Area AE50 from Cave of Chazelles (44 $20^{\prime} \mathrm{N}$ Lat, $4^{\circ} 12^{\prime} \mathrm{E}$ Long), Ardèche. Coll. and subm. by S. Nikitine, CNRS, Paris. Comment: was expected to date settlement just before population of Late Bronze age, but appears to correspond to last period.

Gif-277. Grotte des Crânes, Gard

$5200 \pm 300$

3250 B.C.

Charcoal from N passage of Grotte des Crânes $\left(44^{\circ} 16^{\prime} \mathrm{N}\right.$ Lat, $4^{\circ}$ $08^{\prime}$ E Long), Gard. Comment: this human ossuary is Neolithic. It was thought to be either of Neolithic or Bronze age.

\section{Gif-441. Plateau de Ronzières, Puy-de-Dôme}

$1800 \pm 150$

Charcoal from ruins of Gallo-Roman building, Plateau de Ronzières, (45 $31^{\prime} \mathrm{N}$ Lat, $3^{\circ}$ 07' E Long), Puy de Dôme. Coll. and subm. by G. 
Fournier, Fac. des Lettres de Clermont-Ferrand. Comment: most ceramics of site belong to Late Gallo-Roman period; appears a little older but may be acceptable.

\section{Gif-425. Linard, Haute-Vienne, LIN-10}

$840 \pm 120$

Charcoal from a souterrain, Linard $\left(45^{\circ} 41^{\prime} \mathrm{N}\right.$ Lat, $1^{\circ} 33^{\prime} \mathrm{E}$ Long $)$, Haute-Vienne. Coll. and subm. 1965 by P. Dupuy, Aixe-sur-Vienne. Comment: no archaeologic data, but correct for a medieval souterrain. Cf. Gif-332.

Gif-426. Sereilhac, Haute Vienne, BAI-10

$1320 \pm 120$

Charcoal from a Catalan forge, Sereilhac $\left(45^{\circ} 47^{\prime} \mathrm{N}\right.$ Lat, $1^{\circ} 5^{\prime} \mathrm{E}$ Long), Haute-Vienne. Coll. and subm. 1965 by P. Dupuy. Comment: no archaeologic data, but certainly medieval.

\section{Marcilly-sur-Tille series, Côte d'Or}

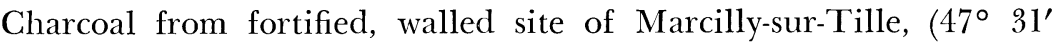
$\mathrm{N}$ Lat, $0^{\circ} 30^{\prime} \mathrm{E}$ Long), Côte d'Or. Coll. and subm. 1963 by E. Planson, Dijon, Côte d'Or.

Gif-230. Marcilly-sur-Tille, M/CN

In layer containing Neolithic artifacts.

Gif-231. Marcilly-sur-Tille, M/TP

Interior of rampart, in stake hole.

Gif-232. Marcilly-sur-Tille, M/FS

At bottom of trench.

Gif-233. Marcilly-sur-Tille, M/CB

$60 \mathrm{~cm}$ beneath surface.

$810 \pm 120$

A.D. 1140

$700 \pm 120$

A.D. 1250

A.D. 1250

$$
700 \pm 120
$$

$510 \pm 120$

A.D. 1440

$2180 \pm 150$

\section{Gif-270. Hauteroche, Côte d'Or}

230 B.C.

Charcoal from burnt material of Gallo-Roman villa, $30 \mathrm{~cm}$ from surface, at Hauteroche ( $47^{\circ} 30^{\prime} \mathrm{N}$ Lat, $0^{\circ} 19^{\prime} \mathrm{E}$ Long), Côte d'Or; assoc. with ancient coins of 2nd and 3rd centuries A.D. Coll. and subm. 1962 by J. Joly, Dir. Antiquités Préhistoriques de Bourgogne, Paris. Comment: date seems too old and probably implies utilization of older wood.

\section{Alésia series, Côte d'Or}

\section{Northern France}

Charcoal from cutting in 2 burnt layers separated by gravel beds, stones, and ground at Alésia (47 $31^{\prime} \mathrm{N}$ Lat, $4^{\circ} 14^{\prime} \mathrm{E}$ Long), Côte d'Or. Coll. 1962 and subm. 1965 by Abbé Joly. 
Gif-267. Alésia I

$1 \mathrm{~m}$ depth.
$1670 \pm 120$

A.D. 280

$1240 \pm 120$

A.D. 710

Gif-268. Alésia II

$1.80 \mathrm{~m}$ depth.

General Comment: level archaeologically undated as no furniture has been found up to the present.

\section{Grotte de la Baume de Gonvillars series, N Jura}

Charcoal and carbonized cereals from habitat in Grotte de la Baume

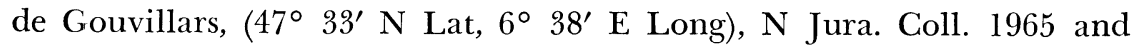
subm. 1966 by P. Petrequin, Lab. Archéol. de Besançon.

\section{Gif-466. Gonvillars, E3V}

$5000 \pm 250$

From Final Bronze II and III level. Comment: following excavation verified an intrusion at this depth of inferior Neolithic levels.

Gif-467. Gonvillars, E5b

$3430 \pm 200$

Late Neolithic level. Comment: corresponds to Michelsberg culture in region. Similar results in Switzerland.

\section{Gif-468. Gonvillars, E6x}

$5380 \pm 250$

Neolithic level. Comment: ceramics with Danubian influence; Late Rössen culture.

\section{Gif-469. Gonvillars G10-XI b}

$6250 \pm 300$

Middle Neolithic level. Comment: seems too old for this Neolithic culture.

4300 B.C.

\section{Gif-360. Tinqueux, Marne}

$3910 \pm 200$

Charcoal from collective tomb, in rock-cut hypogeum, at Tinqueux $\left(49^{\circ} 14^{\prime} \mathrm{W}\right.$ Lat, $3^{\circ} 59^{\prime} \mathrm{E}$ Long), Marne. Coll. 1963 and subm. 1965 by A. Leroi-Gourhan, Centre de Recherches Préhistoriques et Protohistoriques, Paris. Comment: assoc. with Late Neolithic industry of SeineOise-Marne culture (Bailloud and Brezillon, 1968).

Gif-720. Videlles, Seine et Oise

Charcoal from lowest Layer $\mathrm{E}$ of settlement of Les Roches, Loc. 5, Videlles ( $48^{\circ} 25^{\prime} \mathrm{N}$ Lat, $3^{\circ} 35^{\prime} \mathrm{E}$ Long), Seine et Oise. Coll. and subm. 1966 by G. Bailloud, CNRS, Paris. Comment: similar dates for same level: 
$4500 \pm 60:$ GrN-4675, $4500 \pm 50:$ GrN-4676 (Radiocarbon, v. 9, 1967, p. 133); old, if compared to ages of other Late Neolithic sites of Seine-OiseMarne culture, but corresponds in fact to archaic phase of this culture. Layer D, just above Layer E, was dated $2930 \pm 250$ : Gsy-110 (Radiocarbon, v. 8,1966 , p. 132), but it now seems that this level, was contaminated by upper layers.

\section{Pincevent series, Seine et Marne}

Charcoal from hearths of Magdalenian habitation site of Pincevent (48 $23^{\circ} \mathrm{N} \mathrm{Lat,} 2^{\circ} 53^{\prime} \mathrm{E}$ Long), Seine et Marne. Coll. and subm. 1964 by A. Leroi-Gourhan and M. Brezillon.

\section{Gif-349. Pincevent, Y 61, Area 9}

$9840 \pm 350$

$40 \mathrm{~cm}$ depth, under present soil. Comment: date is mean of 2 dates: $9900 \pm 350$ and $9790 \pm 350$ B.P. obtained with same sample.

\section{Gif-358. Pincevent, Hearth III, Habitat 1}

$$
\begin{aligned}
& 12,300 \pm 400 \\
& 10,350 \text { в.C. }
\end{aligned}
$$

General Comment: other measurements made by Louvain and Groningen labs on hearths of same site have given: Lv-291: 10,920 B.P.; Lv-292: 11,610 в.P.; Lv-293: 11,310 в.P. (Radiocarbon, 1969, v. 11, p. 108), GrN4383: 10,760 B.P.

Gif-327. Bulles, Oise

Charcoal from Merovingian cemetery at Bulles $\left(49^{\circ} 28^{\prime} \mathrm{N} \mathrm{Lat}, 2^{\circ} 8^{\prime}\right.$ 30" S Long), Oise. Coll. and subm. 1964 by Y. Legoux. Comment: no significance unless charcoal was found in incinerator where lignite jewelry was burnt?

\section{Gif-339. Bardouville, Seine Maritime}

A.D. 1480

$470 \pm 120$

Bones from sepulchres in Norman sands at Bardouville $\left(49^{\circ} 26^{\prime} \mathrm{N}\right.$ Lat, $0^{\circ} 5 \mathrm{l}^{\prime}$ E Long), Seine Maritime. Coll. by J. Dastugue and subm. 1964 by J. Graindor, Collège de France, Paris. Comment: does not confirm expected Paleolithic age.

\section{Gif-244. La Ferme du Chinchy, $\quad 2830 \pm 150$ Villeneuve-sur-Fère, Aisne \\ 880 B.C.}

Charcoal of hearth at $40 \mathrm{~cm}$ depth, with so-called "Tardenoisian" industry from la Ferme du Chinchy, Villeneuve-sur-Fère $\left(49^{\circ} 39^{\prime} \mathrm{N}\right.$ Lat, $3^{\circ} 22^{\prime}$ E Long), Aisne. Coll. by M. Hinout and subm. 1964 by H. Alimen, CNRS, Bellevue. Comment: not compatible with results for "Tardenoisian" at Coincy, $700 \mathrm{~m}$ farther: $3260 \pm 200$ and $4740 \pm 350$ B.P. (Gif-132 and Gif-133, Radiocarbon, 1966, v. 8, p. 82). Contamination through sands or disturbed site. 
Gif-407. Painting from Musée du Louvre

Fragments of cloth from "Greco-Roman" painting. Subm. by M. Hours, Lab. Mus. du Louvre, Paris. Comment: as expected, painting is forgery.

\section{Western France}

\section{Gif-345. Le Curnic, Guisseney, Finistère,} Hearth Neo. 64

Charcoal from a submerged Neolithic hearth, on beach of the Curnic, Guisseny ( $48^{\circ} 35^{\prime} \mathrm{N}$ Lat, $4^{\circ} 25^{\prime} \mathrm{W}$ Long), Finistère. Coll. and subm. 1965 by C. T. Le Roux and P. R. Giot, Lab. d'Anthropol. Préhistorique, Rennes. Comment: confirms presence on this coast of Early Neolithic population, as shown by Gsy-47B (Radiocarbon, 1966, v. 8, p. 134; Giot, 1961).

\section{Ploudalmezeau, Ile Carn series, Finistère}

Charcoal from megalithic monuments at Ploudalmezeau, Ile Carn ( $48^{\circ} 34^{\prime}$ N Lat, $4^{\circ} 41^{\prime}$ W Long), Finistère. Coll. and subm. 1965, 1966 by P. R. Giot and C. T. Le Roux.

\section{Gif-414. Ile Carn B 3}

$5340 \pm 250$

Central tomb of a megalithic cairn. Comment: result identica GrN-1968: 3270 B.c. for same site.

Gif-1362. Ile Carn, S Carn 2

$5390 \pm 150$

Room in S dolmen. Comment: tomb similar to Gif-414.

Gif-1363. Ile Carn, N Carn

$4840 \pm 150$

2890 B.c.

$\mathrm{N}$ room in $\mathrm{N}$ dolmen. Comment: had a longer frequentation than Carn B and S Carn 2, as was expected.

\section{Gif-393. Abbey of Landevennec, Finistère}

Charcoal from hearth on flagstones, found during excavations in the Landevennec abbey foundations (48 $18^{\circ} 18^{\prime} \mathrm{N}$ Lat, $4^{\circ} 17^{\prime} \mathrm{W}$ Long), Finistère. Coll. and subm. 1965 by M. Ricou, CNRS, Paris. Comment: main part of abbey was built between XIth and XIIIth centuries and it was abandoned towards 1790. Corresponds to one of numerous fires which have destroyed this building.

\section{Le Questel series, Concarneau, Finistère}

Remains of a Gallo-Roman settlement, probably a praefurnium found in a villa at Le Questel, Concarneau $\left(47^{\circ} 53^{\prime} \mathrm{N}\right.$ Lat, $3^{\circ} 45^{\prime} \mathrm{W}$ Long), Finistère (Sanquer, 1965). Coll. 1964 and subm. 1965 by R. Sanquer, Fac. des Lettres de Brest. 
Gif-408. Le Questel

Mortar.

Gif-409. Le Questel

Charcoal.
$3840 \pm 200$

1890 B.C.

$1580 \pm 120$

A.D. 370

General Comment: assoc. with sigillated ceramics. It may be seen from Gif-408 that lime used to make the mortar was incompletely decarbonized. Gif-409 should date reutilization of site after destruction by the Saxons ca. 250 to 300 A.D.

Gif-346. Pendreff, Commana, Finistère

$2500 \pm 180$

Charcoal from hearth in Rooms III, IV, of an Iron age souterrain at Pendreff, Commana (Le Roux and Giot, 1966) $\left(48^{\circ} 25^{\prime} 12^{\prime \prime} \mathrm{N}\right.$ Lat, $3^{\circ}$ $57^{\prime} 46^{\prime \prime}$ W Long), Finistère. Coll. and subm. 1965 by P. R. Giot and C. T. Le Roux. Comment: as for many of Armorican souterrains, some centuries older in regard to ceramics, which here could be expected to date from 350 to 300 B.C. (Giot, 1966, 1967).

\section{Gif-716. Moulin de la Rive, Locquirec, Finistère}

$$
1900 \pm 100
$$

Charcoal from Iron age habitat in sand hill, Moulin de la Rive, Locquirec ( $48^{\circ} 41^{\prime} \mathrm{N}$ Lat, $3^{\circ} 42^{\prime} \mathrm{W}$ Long), Finistère. Coll. and subm. by P. R. Giot and C. T. Le Roux. Comment: perhaps 100 yr too young but fits well with La Tene III age of ceramics (Giot, 1968).

\section{Gif-481. Plouégat, Moysan, N Finistère}

$$
2480 \pm 110
$$

530 в.C.

Charcoal from Iron age souterrain, Plouégat, Moysan, Bellevue $\left(48^{\circ}\right.$ $34^{\prime}$ N Lat, 3० $37^{\prime}$ W Long), N Finistère. Coll. and subm. 1966 by P. R. Giot. Comment: a few centuries older than expected; archaeologic material might be dated to 150 or 100 B.c. (Giot et al., 1965).

\section{Gif-715. Fossé de Catuélan, Erquy, Côtes du Nord}

$2500 \pm 110$

550 в.C.

Charcoal from Late Bronze age oppidum at Erquy $\left(48^{\circ} 39^{\prime} \mathrm{N}\right.$ Lat, $2^{\circ} 28^{\prime}$ W Long), Côtes du Nord. Coll. and subm. 1966 by P. R. Giot and C. T. Le Roux. Comment: corresponds to expected age.

\section{Gif-344. Crec'h Quillé, Saint-Quay, Perros, $\quad 3740 \pm 200$ Côtes du Nord \\ 1790 B.C.}

Charcoal from blocking of lateral entrance grave Crec'h Quillé, Saint-Quay-Perros (48 47' N Lat, $3^{\circ} 23^{\prime}$ W Long), Côtes du Nord (L'Helgouach, 1967). Coll. and subm. 1964 by J. L'Helgouach, Fac. des Sci. de Rennes. Comment: slightly younger than expected, dates final filling in of tomb, cf. Gif-197C (Radiocarbon, 1966, v. 8, p. 74, Gif-II) (Giot, 1965). 
Gif-487. Plouasme, Le Bourg, Côtes du Nord

A.D. 970

$980 \pm 100$

Wood found under a Middle Age mound at Plouasme, Le Bourg (53 $76^{\prime} \mathrm{N}$ Lat, $4^{\circ} 85^{\prime} \mathrm{W}$ Long), Côtes du Nord. Coll. and subm. 1966 by M. Monier, Dinan, Côtes du Nord.

\section{Gif-718. Saint Goueno, Côtes du Nord}

$1030 \pm 100$

Charcoal from a level of fire of the oppidum of Cap Barré de la Parentelaye, Saint-Goueno $\left(48^{\circ} 14^{\prime} \mathrm{N}\right.$ Lat, $2^{\circ} 33^{\prime} \mathrm{W}$ Long), Côtes du Nord. Coll. and subm. 1966 by C. T. Le Roux, P. R. Giot. Comment: dates this important protohistoric oppidum.

\section{Gif-717. Guernehué, Monterblanc, Morbihan \\ 230 B.C.}

$2180 \pm 100$

Charcoal from Iron age souterrain, Guernehué, Monterblanc $\left(47^{\circ}\right.$ $45^{\prime} \mathrm{N}$ Lat, $2^{\circ} 40^{\prime} \mathrm{W}$ Long), Morbihan. Coll. and subm. 1966 by J. C. Lecornec, Vannes. Comment: fits with assoc. ceramics.

\section{Gif-7 19. La Grée Basse, Monteneuf, Morbihan}

$2850 \pm 110$

900 B.C.

Charcoal from grave incineration at La Grée Basse, Monteneuf $\left(47^{\circ} 53^{\prime} \mathrm{N}\right.$ Lat, $2^{\circ} 12^{\prime} \mathrm{W}$ Long), Morbihan. Coll. and subm. 1966 by M. Orhan, Ploërmel, Morbihan. Comment: no archaeologic clue to date site.

\section{Goërem series, Gâvres, Morbihan}

Charcoal from angled passage grave of Goërem, Gâvres $\left(47^{\circ} 41^{\prime}\right.$ 47" N Lat, $30^{\circ} 21^{\prime}$ 12" W Long), Morbihan. Coll. and subm. 1964 by J. L'Helgouach.

Gif-329. Goërem I, $\mathbf{A W}{ }^{6}$

$3860 \pm 200$

Comment: dates Chalcolithic (Bell-Beaker) frequentation of site.

\section{Gif-330. Goërem II, $\mathbf{A W ^ { 4 } - \mathbf { A W }} \mathbf{W}^{6}$}

$2620 \pm 200$

Comment: indicates Iron age intrusion.

670 B.C.

\section{Gif-768. Goërem III}

$4100 \pm 140$

2150 B.c.

In room of grave, under broken pavement. Comment: presence of so-called ceramics of Kerugou.

\section{Gif-769. Goërem IV}

Upper hearth in room. Comment: hearth in relation with an attempt to penetrate into the sepulchre. 


\section{Gif-1148. Goërem IV b}

From deepest level of filling of the room, separated from Goërem IV by stones. Comment: important layer with "Kerigou" and campaniform ceramics.

\section{Gif-482. Saint-Evarzec, $S$ Finistère}

Charcoal from Bronze Age barrow, Saint-Evarzec, Kerhuel $\left(47^{\circ} 56^{\prime}\right.$ $\mathrm{N}$ Lat, $4^{\circ}$ 02' W Long), S Finistère (Le Roux, 1966). Coll. 1965 and subm. 1966 by G. T. Le Roux. Comment: slightly younger date was expected; dates beginning of Middle Bronze age.

\section{Gif-410. Le Boucaud, Préfailles, Loire Atlantique}

Charcoal from brickworks of Late Bronze age, in a shist clift at Le Boucaud, Préfailles $\left(47^{\circ} 8^{\prime} \mathrm{N}\right.$ Lat, $2^{\circ} 14^{\prime} \mathrm{W}$ Long), Loire Atlantique, (Tessier and Gouletquer, 1966). Coll. and subm. 1965 by M. Tessier, Tharon, Loire Atlantique. Comment: agrees well with well-dated habitation site of Late Bronze age, at Saint-Brévin l'Océan, Gif-193 (Radiocarbon, v. 8, 1966, p. 80).

\section{La Plaine-sur-Mer series, Loire Atlantique}

Charcoal from sites with "augets," pottery salt-pans of Iron age, at La Plaine-sur-Mer, Loire Atlantique (Gouletquer et al., 1967-1969). Coll. and subm. 1965 by P. L. Gouletquer, Lab. d'Anthropol. Préhistorique, Rennes.

Gif-411. La Frenelle, La Plaine-sur-Mer $\left(48^{\circ} 8^{\prime} \mathrm{N}\right.$ Lat, $2^{\circ} 13^{\prime} \mathrm{W}$ Long)

Gif-412. La Tarra, La Plaine-sur-Mer $\left(47^{\circ} 10^{\prime} \mathrm{N}\right.$ Lat, $2^{\circ} 15^{\prime} \mathrm{W}$ Long)

General Comment: Late Iron age date on archaeologic ground. All salt industry sites of this type found in different regions of Brittany have similar characteristics, i.e., often near the sea, they contain a lot of "augets" often broken, supposedly, while withdrawing salt from mould.

\section{Gif-413. Monzenil, Vendée}

$1840 \pm 150$

Charcoal from salt industry site at Monzenil $\left(49^{\circ} 29^{\prime} \mathrm{N}\right.$ Lat, $1^{\circ}$ 00’ W Long), Vendée. Coll. and subm. 1965 by P. L. Gouletquer.

\section{Semussac series, Charente Maritime}

Shells from Peu-Richardien camp of Semussac $\left(45^{\circ} 36^{\prime} \mathrm{N}\right.$ Lat, $0^{\circ} 55^{\prime}$ W Long), Charente Maritime (Mohen, 1967). Coll. and subm. 1966 by J. P. Mohen, Merignac, Gironde. 
Gif-474. Semussac, Level 1

$4690 \pm 250$

Lowest level, $1.35 \mathrm{~m}$ depth. Comment: expected age: ca. 2700 в.c.

\section{Gif-475. Semussac, Level 3}

$4250 \pm 250$

Upper level. Comment: expected age: ca. 2500 B.c.

2300 B.c.

\section{La Garenne de Saint-Hippolyte series, Charente Maritime}

Samples from Peu-Richard site of La Garenne de Saint-Hippolyte $\left(45^{\circ} 54^{\prime} \mathrm{N}\right.$ Lat, $0^{\circ} 50^{\prime} \mathrm{W}$ Long), Charente Maritime (Gabet and Massaud, 1965, 1966). Coll. 1963 by C. Gabet; subm. 1964, 1965 by J. Massaud, Angoulême.

Gif-313. La Garenne de Saint-Hippolyte, 1964

Charcoal from hearth at bottom of chamber.

Gif-417. La Garenne de Saint-Hippolyte, 1965

$4560 \pm 250$

Patellae from archaeologic layer.

General Comment: shows antiquity of this culture in this place with regard to more continental sites.

$$
\text { II. AFRICA }
$$

Gif-306. R'Fana, R.F. 1, Tebessa, Bône, Algérie

Charcoal from snailery of Upper Capsian at R'Fana, Tebessa, Bône (35 $21^{\prime} \mathrm{N}$ Lat, $8^{\circ} 06^{\prime} \mathrm{E}$ Long), Algeria. Coll. and subm. 1963 by G. Camps, Centre de Recherches Anthropol. Préhistoriques et Ethnog. Mus. du Bardo, Alger.

\section{Columnata series, Tiaret}

Columnata ( $35^{\circ} 32^{\prime} 30^{\prime \prime} \mathrm{N}$ Lat, $1^{\circ} 30^{\prime} 55^{\prime \prime} \mathrm{E}$ Long), Tiaret, in central part of Maghreb, is key site for study of Epipalaeolithic and Capsian ages in N Africa. Charcoal coll. 1962 and subm. 1965 by G. Camps.

Gif-307. Columnata, 100 to $130 \mathrm{~cm}$ depth

Gif-308. Columnata, 160 to $200 \mathrm{~cm}$ depth

Gif-309. Columnata, 200 to $230 \mathrm{~cm}$ depth
$5250 \pm 250$ 3300 B.c.

$6850 \pm 300$ 4900 B.c.

$6340 \pm 300$ 4390 в.c.

General Comment: show passage from Upper Capsian (Gif-308 and Gif-309) to a Neolithic with Capsian and Mediterranean traditions. The 130 to 160 level dated 6800 в.P. by MC-154 (Radiocarbon, 1969, v. 11, p. 126; Camps et al., 1968). 
Gif-438. Hassi-Mouilah E 10, Ouargla, Algeria 3330 B.C.

Charcoal from Neolithic hearth, Hassi Mouilah E 10, Ouargla $\left(32^{\circ}\right.$ $00^{\prime} \mathrm{N}$ Lat, $5^{\circ} 16^{\prime} \mathrm{E}$ Long). Coll. and subm. 1965 by M. Trecolle, Ouargla. Comment: agrees very well with other dates obtained for the Neolithic age of Caspian tradition.

\section{Gif-365. Hassi Manda, Algeria}

$6330 \pm 300$

Charcoal from Neolithic site of Capsian tradition at Hassi Manda on the bank of Es-Roui Erg, NW Sahara (29 $15^{\prime} \mathrm{N}$ Lat, $2^{\circ} 30^{\prime} \mathrm{W}$ Long); nondetermined industry; remains of Sudanese fauna. Coll. and subm. 1965 by J. Mateu, CNRS, Paris.

\section{Gif-366. Foum Seida, Algeria}

$4930 \pm 250$

Charcoal from Neolithic site of Foum Seita, Beni-Abbès $\left(30^{\circ} 11^{\prime}\right.$ $\mathrm{N}$ Lat, $2^{\circ} 14^{\prime} \mathrm{W}$ Long). Coll. and subm. 1965 by J. Mateu.

\section{Gif-461. Tipasa, Algeria, TPS 1}

$2300 \pm 150$

Charcoal from hearth in $\mathrm{W}$ necropole, at Tipasa $\left(36^{\circ} 32^{\prime} \mathrm{N}\right.$ Lat, $2^{\circ}$ E Long), Algeria. Coll. and subm. 1966 by G. Camps. Comment: assoc. with Punic ceramics. Dates Punic settlement.

\section{Gif-462. Medjez II, Setif, E Algeria, MJ21}

$6620 \pm 300$

Charcoal from ashy level, in a snailery, at Medjez $\left(36^{\circ} 11^{\prime} \mathrm{N}\right.$ Lat, $5^{\circ} 42^{\prime}$ E Long), E Algeria. Coll. and subm. 1966 by G. Camps. Comment: industry of this site has been used to define an Upper Capsian facies.

\section{Gif-463. "Bou-Sfer" W Algeria, ESC-1}

$6680 \pm 300$

4730 B.C.

Charcoal from hearth in ashy archaeologic level, of littoral site of Bou-Sfer $\left(35^{\circ} 40^{\prime} \mathrm{N}\right.$ Lat, $1^{\circ} 10^{\prime} \mathrm{W}$ Long), W coast of Algeria. Coll. 1960 by G. Vuillemot and subm. 1966 by G. Camps. Comment: site wellknown for its Neolithic ceramics; agrees with dates obtained for similar sites in Spain and Italy.

\section{Gif-464. Tamanrasset, Amekni, Hoggar, AMK $1 \quad 350$ B.c.}

Charcoal from hearth in upper archaeologic level, 30 to $60 \mathrm{~cm}$ depth, in site of Amekni, Tamanrasset (23 $13^{\prime} \mathrm{N}$ Lat, $5^{\circ} 13^{\prime}$ E Long), Hoggar. Coll. 1965 and subm. 1966 by G. Camps. Comment: assoc. with Neolithic ceramics of Sudan tradition. 


\section{Gif-357. Tamanrasset, Hoggar}

Charcoal from the Neolithic burial site near Tamanrasset $\left(23^{\circ} 10^{\prime}\right.$ $\mathrm{N}$ Lat, $5^{\circ} 35^{\prime}$ E Long), Hoggar. Coll. and subm. 1964 by G. Camps. Comment: found with 2 Negroid skeletons accompagnied by rough ceramics and tools. Too young to date site, perhaps dates re-utilization.

\section{Hoggar series}

Samples from Pre-Islamic graves, in the Hoggar. Coll. 1910 by P. Reygasse, kept in Mus. du Bardo, Algiers and subm. 1966 by M. C. Chamla, Inst. de Paléontol. Humaine, CNRS, Paris.

Gif-700. Silet, No. 3

$$
420 \pm 100
$$

Charcoal from a stone-barrow at Silet $\left(23^{\circ} \mathrm{N}\right.$ Lat, $5^{\circ} \mathrm{E}$ Long).

Gif-701. Tit, No. 4

$$
650 \pm 100
$$

Piece of cloth from a stone-barrow at Tit $\left(23^{\circ} \mathrm{N}\right.$ Lat, $5^{\circ} \mathrm{E}$ Long).

Gif-702. Tit, No. 68

$680 \pm 100$

Piece of cloth from a stone-barrow, at Tit $\left(23^{\circ} \mathrm{N}\right.$ Lat, $5^{\circ} \mathrm{E}$ Long $)$.

\section{Gif-703. Coralès I}

$440 \pm 100$

Charcoal from hearth in a sand-hill $\left(36^{\circ} \mathrm{N}\right.$ Lat, $1^{\circ} \mathrm{W}$ Long).

General Comment: samples date occupation of the Hoggar during protohistoric period.

\section{Gif-465. Tamanrasset, Abouleg I, Hoggar}

$4600 \pm 250$

Charcoal from Level I, Abouleg, Tamanrasset $\left(22^{\circ} 50^{\prime} \mathrm{N}\right.$ Lat, $5^{\circ} 31^{\prime}$ E Long), Hoggar. Coll. by J. P. Maitre and subm. 1966 by G. Camps. Comment: compare with other dates obtained for Levels I and II: 3140 \pm 80 and $4190 \pm 80$ в.c. (UW-88 and UW-89). (Camps et al., 1968).

\section{Gif-375. Amded Oued, W Hoggar}

$$
2160 \pm 150
$$

Fragment of elephant-tusk found at surface of Amded Oued 150 $\mathrm{km} \mathrm{W}$ of Tamanrasset $\left(22^{\circ} 35^{\prime} \mathrm{N}\right.$ Lat, $3^{\circ} 55^{\prime} \mathrm{E}$ Long). Coll. and 1965 by C. Arambourg. Comment: this result, much younger than expected, is not significant of formation of regs from ancient flow of Amded Oued. 


\section{Ennedi series, Tchad}

In $\mathrm{S}$ W Massif de l'Ennedi, at the limit of the Tchad, more than 500 sites with ruspestral paintings were counted by 1956-1957 CNRS expedition. The "Pebble culture", well-known in Tibesti, is absent in these sites; the lithic industry is too poor to characterize different periods of Neolithic age, but, the ceramic industry is very rich and various. Charcoals coll. 1957 and subm. 1965 by Bailloud, CNRS, Paris.

Gif-351. Ennedi, Delebo, 2-III

$7200 \pm 300$

( $17^{\circ} 12^{\prime} \mathrm{N}$ Lat, $21^{\circ} 15^{\prime} \mathrm{E}$ Long), accompanied by wavy line pottery; dates beginning of Neolithic.

Gif-352. Ennedi, Delebo, 2 II

$6900 \pm 300$

$\left(17^{\circ} 12^{\prime} \mathrm{N}\right.$ Lat, $21^{\circ} 15^{\prime} \mathrm{E}$ Long), ceramics show affinity with Sudanese civilization of Esh Shaheinab.

\section{Gif-353. Ennedi, Tenebyela}

$400 \pm 120$

( $17^{\circ} 30^{\prime} \mathrm{N}$ Lat, $21^{\circ} 37^{\prime} \mathrm{E}$ Long), same archaeologic context as Gif352. Comment: found very near surface: this explains intrusion of recent charcoal into archaeologic layer.

\section{Gif-354. Ennedi, Cobé V}

$5000 \pm 250$

( $17^{\circ} 19^{\prime} \mathrm{N} \mathrm{Lat}$, $21^{\circ} 40^{\prime} \mathrm{E}$ Long), ceramics of Hohou type from "Middle Neolithic" rougher and simpler decoration than ceramics of 1st Neolithic age.

General Comment: coherent with other dates obtained for Saharian Neolithic.

\section{Tchad series}

Charcoal from 3 sites from ancient and middle phases of Sao culture in the Tchad. They are 3 of the 200 sites presently known, between 20 and $30 \mathrm{~km} \mathrm{~N}$ Fort-Lamy (12 $10^{\prime} \mathrm{N}$ Lat, $14^{\circ} 59^{\prime} \mathrm{E}$ Long), Tchad, habitation mounds corresponding to remains of ancient villages. Coll. 1963 and subm. 1965 by J. P. Lebeuf, Lab. d'Ethnol. et d'Archéol. Tchadienne, Paris.

Gif-428. Mdaga, VII, $1.10 \mathrm{~m}$ depth

Modern

Gif-429. Mdaga, VII, $1.50 \mathrm{~m}$ depth

A.D. 1620

Gif-432. Amkoundjo, 0.30 to $0.60 \mathrm{~m}$ depth

$$
1980 \pm 180
$$

30 B.c.

Gif-433. Amkoundjo, $1 \mathrm{~m}$ depth 
Gif-435. Amkoundjo, $2.60 \mathrm{~m}$ depth

\section{Gif-436. Mound of Messo, 2 to $2.20 \mathrm{~m}$ depth}

\section{Gif-374. Madaouela, Nigeria}

Charcoal from Neolithic surface site, at Madaouela $\left(18^{\circ} 40^{\prime} \mathrm{N}\right.$ Lat, $7^{\circ} 35^{\prime}$ E Long), ca. $200 \mathrm{~km} \mathrm{~N}$, NW Agadès, Nigeria. Archaeologic layer, $30 \mathrm{~cm}$ depth gave abundant culinary remains (bones, shells, fish bones, pieces of pottery, stone tools, and arrow heads). Site found by uranium prospectors, F. Chantret and R. de Bayle des Hermens, 1968. Coll. and subm. by M. Chantret, Comm. à l'Energie Atomique, Fontenay-auxRoses, France. Comment: agrees with other dates for Neolithic period in Sahara (Chantret and Bayle des Hermens, 1968).

\section{Tiebala series, Mali, W Africa}

Charcoal from detritus pit on side of the "Middle Ages" Site F, 3 $\mathrm{km} \mathrm{N}$ Tiebala $\left(13^{\circ} 40^{\prime} 40^{\prime \prime} \mathrm{N}\right.$ Lat, $5^{\circ} 24^{\prime} 20^{\prime \prime} \mathrm{W}$ Long), Mali. Site F is ancient mound of dwelling in which were found ceramics of unknown kind. Coll. and subm. 1964 by A. Gallay, CNRS, Paris.

\section{Gif-383. Tiebala, FII 1, $0.5 \mathrm{~m}$ depth}

$1440 \pm 120$

\section{Gif-384. Tiebala, FII 2, 1 m depth} A.D. 510

$$
1400 \pm 120
$$

A.D. 550

General Comment: local traditions mention that site was occupied in 13th century. Dates are important references for establishing absolute chronology by ceramics in W Africa.

\section{Mirgissa series, Wadi Halfa, Sudan}

Charcoal from Egyptian town of Mirgissa $\left(21^{\circ} 55^{\prime} \mathrm{N}\right.$ Lat, $31^{\circ} 20^{\prime}$ E Long), dist. of Wadi Halfa, Rep. of Sudan. Mirgissa is 1 of ancient sites of upper valley of Nile which is now submerged, since construction of Aswan dam. Coll. 1963 and subm. 1964 by J. Vercoutter, Fac. des Sci. de Lille.

Gif-295. Mirgissa M.F. 1

$2925 \pm 180$

Charcoal at interior of bricks made of dried silt found in fortress of Mirgissa.

Gif-296. Mirgissa M. VII. 2

$3780 \pm 200$

Similar to Gif-295. 
Gif-297. Mirgissa, M.I., BT. 1

Charcoal from hearth covered by Eolian sand.

Gif-185. Majunga, Madagascar

$3780 \pm 200$

1830 B.c.

Wood from peat bog at Majunga (15 $40^{\prime} \mathrm{S}$ Lat, $46^{\circ} 20^{\prime} \mathrm{E}$ Long), Madagascar. Peat bog is also fossil mammalian site. Coll. 1962 by M. Mahé and subm. 1964 by J. P. Lehman, Inst. de Paléontol., Paris. Comment: date seems too young for Malagasy subfossils.

\section{OTHER COUNTRIES}

\section{Hang Gon, South Viet Nam}

Carbonized deposit on potsherds of burial urns, from site of Hang Gon, near Xuan Loc $\left(10^{\circ} 48^{\prime} \mathrm{N}\right.$ Lat, $107^{\circ} 15^{\prime} \mathrm{E}$ Long), S Viet Nam. Coll. and subm. 1965 by E. Saurin, Fac. des Sci. de Saigon. Comment: dates "Sa Huynh" culture. Identical results for charcoal found outside and inside similar burial urns in same place (MC-61, MC-62, Radiocarbon, 1966, v. 8, p. 290).

\section{Biskupin series, ZNIN, Poland}

The ancient town of Biskupin, Znin ( $52^{\circ} 51^{\prime} \mathrm{N}$ Lat, $17^{\circ} 41^{\prime} \mathrm{E}$ Long), Poland is considered as the birthplace of the Lusatian civilization; there is an important controversy about the possible Lusatian origin of Slav people. During the Iron age, a fortified town with ramparts $6 \mathrm{~m}$ high, $463 \mathrm{~m}$ long, was built in Biskupin. After destruction of the city, a new one was built, according to the same general survey. Samples are pieces of wood from ancient city. Subm. by Z. A. Rajewski, Panstwowe Archaeol., Warsaw.

\section{Gif-224. Biskupin, Area 112}

$1635 \pm 150$

Wood from fortifications. Coll. 1959, subm. 1963. Comment: age too young; possible mixing of materials from the 2 towns.

Gif-492. Biskupin, fortification

Subm. 1966.

Gif-493. Biskupin, A2 4c, VII

$85 \mathrm{~cm}$ depth, subm. 1966.

Gif-494. Biskupin, 1st settlement

Subm. 1966. 
Gif-495. Biskupin, later settlement

560 B.C.

General Comment: places period of settlement 750 to 550 B.C., during Halstatt C, when it supposedly was of Halstatt D, 550 to 400 B.c. Duration for the 2 settlements, ca. $200 \mathrm{yr}$, agrees well with archaeologic data.

\section{Gif-66. Zàmeček, Slovakia}

$2350 \pm 150$

Burnt corn from habitat from Late Bronze age $\mathrm{A}^{2}$, Zàmeček, Nitriansky Hràdec, Slovakia. Coll. and subm. by P. R. Giot. Comment: too young.

\section{Mallia series, Kriti Island}

Charcoal from Mallia (35 42' N Lat, $25^{\circ} 30^{\prime}$ E Long), Kriti I. Coll. 1963 and subm. 1964 by O. Pelon, Ecole Française d'Athènes.

\section{Gif-254. Mallia I}

$4030 \pm 300$

Burnt layer, $1.50 \mathrm{~m}$ depth, $\mathrm{E}$ zone of town. Comment: agrees with assoc. ceramics which indicate that level of occupation is latest before construction of palace of Mallia between 2100 and 1900 B.c.

\section{Gif-255. Mallia II}

$3470 \pm 250$

$2.10 \mathrm{~m}$ depth, Sq. $\mathrm{A}^{3}$, W zone of town. Comment: level corresponds to mean Minoan III in chronology of Knossos.

\section{Gif-256. Mallia III}

$3200 \pm 250$

$1.20 \mathrm{~m}$ depth, Sq. A4, $4 \mathrm{~m}$ from Mallia II. Comment: too young.

\section{Gif-470. Temple of Bêl, Palmyre, Syria}

$3420 \pm 350$

Bones of animals, $1.20 \mathrm{~m}$ depth in a tell, Temple of Bêl, Palmyre (34 $35^{\circ}$ N Lat, $38^{\circ} 17^{\prime}$ E Long), Syria. Coll. 1965 and subm. 1966 by R. du Mesnil du Buisson, CNRS, Paris. Comment: coherent with stratigraphy of tell, which appears to go from 12th century в.c. to 22nd century B.C. Bones from lower levels had no more organic matter and could not be dated.

\section{Tureng Tepe series, Gorgan, Iran}

Charcoal from Tureng Tepe $\left(36^{\circ} 55^{\prime} \mathrm{N}\right.$ Lat, 54 $35^{\circ} \mathrm{E}$ Long), in the steppe, SE Capsian sea, Iran. Coll. 1963 and subm. 1964 by J. Deshays, Fac. des Lettres et Sci. Humaines, Lyon.

\section{Gif-301. Tureng Tepe 2}

Remains of wooden post which supported roof of house built of raw bricks, House B, $5 \mathrm{~m}$ depth. Comment: over House B, another level 
of destruction with House A, corresponding to Tureng Tepe II B, a civilization similar to Tepe Hissar II B in Mesopotamia. Above House A, a different civilization appears, with construction in pisé, which had commercial relations with Mesopotamia; time of 1st dynasty of Our, ca. 2500 to 2400 B.c.

\section{Gif-302. Tureng Tepe 4}

$4090 \pm 250$

From House F, $6.50 \mathrm{~m}$ depth. Comment: one of deepest levels of Tureng Tepe II B. This level is surely older than Tureng Tepe 2. Date is too young.

\section{Gif-485. Tureng Tepe $X$}

$3970 \pm 200$

From burning level, $3.50 \mathrm{~m}$ depth. Comment: corresponds to end of so-called Hissar III B civilization. Archaeologically dated, 4000 в.P.

\section{Suse series, Iran}

Ashes mixed with remains of bones from Suse near by Castle of Darius (32 $12^{\prime} \mathrm{N}$ Lat, $48^{\circ} 20^{\prime} \mathrm{E}$ Long), Iran. Coll. and subm. by $\mathrm{M}$. Ghirschman, Acad. des Inscriptions et Belles Lettres, Paris.

Gif-180. Suse C, No. 1

$3175 \pm 250$

On Suse Hill, called "Acropolis", from a habitation level. Supposed to date from end of 4 th millennium B.c.

Gif-182. Suse, A-XIII, No. 3, Loc. 152

$3920 \pm 250$

From excavation in elamite part of town; dated from middle of 2nd millennium B.c. by cuneiform writing tablets.

Gif-183. Suse, A-XIII, No. 4, Loc. 117

$1750 \pm 150$

Similar to Gif-182.

Gif-184. Suse, A-XIII, No. 5

$2730 \pm 200$

Similar to Gif-182.

General Comment: only correct date is Gif-182. Such a disagreement can only be explained by errors of sampling.

\section{Gif-473. Jerusalem}

$300 \pm 100$

Bark and alburn of olive tree from hill dominating Garden of Olives at Jerusalem ( $31^{\circ} 47^{\prime} \mathrm{N}$ Lat, $35^{\circ} 14^{\prime} \mathrm{W}$ Long). Coll. and subm. 1965 by J. Chabanon. Comment: not so old as was hoped. 
REFERENCES

Bailloud, G. and Brézillon, M., 1968, l'Hypogée de l'Homme-Mort à Tinqueux (Marne): Soc. Prehis. Française Bull., v. 65, p. 479-504.

Bouchud, J., 1964, Découverte d'un crâne de renne fossile dans la grotte Bernard près de Foix, Ariège: Acad. sci. (Paris) Comptes rendus, v. 258, p. 4305-4307.

Camps, G., Delibrias, G., et Thommeret, J., 1968, Chronologie absolue et succession des civilisations préhistoriques dans le Nord de l'Afrique: Libyca, v. XVI, p. 9-28.

Chantret, F. and R. de Bayle des Hermens, 1968, Le gisement préhistorique de Madaouéla, République du Niger. Une nouvelle datation pour le Néolithique Saharien: Soc. Préhist. Française Bull., v. 65, p. 623-628.

Costantini, G., 1965, La grotte 1 des Cascades, Commune de Creissels (Aveyron): Soc. Préhist. Française Bull., v. 62, p. 649-666.

Coursaget, J. and Le Run, J., 1966, Gif-sur-Yvette natural radiocarbon measurements I: Radiocarbon, v. 8, p. 128-141.

Courtin, J., 1963, Cahiers ligures de Préhistoire et d'Archéologie, v. 12, p. 214-215.

Delibrias, G., Guillier, M. T., and Labeyrie, J., 1966, Gif natural radiocarbon measurements II: Radiocarbon, v. 8, p. 74-95.

Gabet, C. and Massaud, J., 1965, Le gisement Peu-Richardien de la Garenne 2, Commune de Saint-Hippolyte (Charente Maritime): Soc. Préhist. Française Bull., v. 62 , p. $159-195$.

1966, Datation ${ }^{14} \mathrm{C}$ du Peu-Richardien de la Garenne: Soc. Préhist. Francaise Bull., v. 96.

Gilot, E., 1969, Louvain radiocarbon measurements VII: Radiocarbon, v. 11, p. 106-111.

Giot, P. R., Chronique des datations radiocarbone armoricaines: Annales de Bretagne 1961 , v. 68 , p. $21-24 ; 1965$, v. 72 , p. $133-147 ; 1966$, v. 73 , p. $124-129 ; 1967$, v. 74 , p. $150-153 ; 1968$, v. $75,153-164$.

Giot, P. R., Gouletquer, P. L., et Le Roux, C. T., 1965, Fouille d'un souterrain de l'Age de Fer à Bellevue, en Plouegat-Moysan (Finistère): Annales de Bretagne, v. 72 , p. $115-132$

Gouletquer, P. L. et al., Etudes sur les briquetages: Annales de Bretagne, 1967, v. 74, p. $99-119 ; 1968$, v. 75 , p. $117-148 ; 1969$, v. 76 , p. $119-147$.

Grosjean, R., 1966, Le complexe torréen fortifié du Castello d'Alo (Commune de Bilia, Corse): Soc. Préhist. Française Bull., v. 58.

1967, Premiers travaux et études sur le groupe d'alignement de Palaggiu (Corse): Soc. Préhist. Française Bull., v. 22.

Lagrand, C. H., 1962, Cahiers ligures de Préhistoire et d'Archéologie, v. 11, p. 263.

Leroi-Gourhan, A. and Brézillon, M., 1966, L'habitation magdalenienne $\mathrm{n}^{\circ} \mathrm{l}^{\mathrm{P}}$ de Pincevent, près Montereau: Gallia, Préhistoire, v. 9, $n^{\circ} 2$, p. 263-371.

Le Roux, C. T., 1966, Fouille d'un tumulus de l'Age du Bronze à Kerhuel en SaintEvarec (Finistère): Annales de Bretagne, v. 73, p. 13-31.

Le Roux, G. T. and Giot, P. R., 1965, Fouille d'un souterrain de l'Age de Fer à Pendreff en Commana: Annales de Bretagne, v. 72, p. 95-113.

L'Helgouach, J., 1967, La sépulture mégalithique à entrée latérale de Crec'h-Quillé en Saint-Quay-Perros (Côtes du Nord): Soc. Préhist. Française Bull., v. 64, p. 559-698.

Maury, J. and Lacas, M., 1965, Un gisement sauveterrien sur les Grandes Causses: l'abri des Salzets (Commune de Mostuéjouls, Averyron): Soc. Préhist. Française Bull., v. 67-70.

1965, Un gisement mésolithique inédit sur les Grandes Causses; l'abri II de Puechmargues (Commune de La Roque Sainte-Marguerite, Aveyron): Soc. Préhist. Française Bull., v. 251-255.

Mohen, J. P., 1967, Notes préliminaires à propos des fouilles du camp néolithique de "chez Reine" (Commune de Semussac, Charente Maritime): Soc. Préhist. Francaise Bull., v. 64, p. 469-482.

Montjardin, R., 1967, Première datation du chalcolithique de la Basse-Ardèche: Soc. Préhist. Française Bull., v. 71, p. 11.

Paccard, M., 1965, Un gisement mésolithique en péril: la station de Grand'Marie à Méthamis (Vaucluse): Soc. Préhist. Francaise, Bull., v. 103-107.

1966, Nouvelles découvertes à Gramari (Méthamis, Vaucluse): Soc. Préhist. Française Bull., v. 150-151.

Sanquer, R., 1965, L'établissement Gallo-Romain du Questel en Concarneau: Annales de Bretagne, v. 72, p. 157-173.

Soutou, A., 1966, La Grotte sanctuaire de Sargel: OGAM, v. 18, no. 103-104, p. 1-16.

Tessier, M. and Gouletquer, P. L., 1966, Etudes sur les briquetages: Annales de Bretagne, v. 73 , p. $55-118$ 